\title{
Spatial disorders and computational cures
}

\author{
A. C. Steven* and E. Kocsis \\ Laboratory of Structural Biology Research, National Institute for Arthritis, Musculoskeletal, and Skin Diseases, \\ National Institutes of Health, Bethesda, MD 20892, USA \\ M. Unser \\ Biomedical Engineering and Instrumentation Program, National Center for Research Resources, \\ National Institutes of Health, Bethesda, MD 20892, USA \\ and B. L. Trus \\ Computer Systems Laboratory, Division of Computer Research and Technology, National Institutes of \\ Health, Bethesda, MD 20892, USA
}

\begin{abstract}
Image averaging provides a powerful method for enhancing the yield of interpretable information from electron micrographs of biological macromolecules. However, as originally conceived, the full benefit of averaging is achieved only with perfectly ordered two-dimensional crystals. More recent developments, reviewed here, allow one to rectify disordered lattices, straighten randomly bent filaments, and combine multiple images of free-standing particles, thus extending the advantages of image averaging to virtually every class of macromolecular specimen.
\end{abstract}

Keywords: Digital image processing; biological electron microscopy; spatial disorder; protein crystals; curvilinear coordinates; correlation averaging; multivariate statistics

\section{Introduction}

Image averaging is one of the most basic, widely used, and ultimately informative, image processing operations applied to biological electron micrographs. Averaging results in improved signal-to-noise ratios, producing images that are better defined, and thus more readily interpretable, particularly in the high spatial frequency range where their finest details are delineated. The basic premise underlying image averaging is that, in a set of different images of molecules of the same kind, the signal component is common to all, whereas the noise varies randomly from image to image: thus, averaging should reinforce the signal and suppress the noise ${ }^{1}$. In practice, the assumption that biological electron micrographs are compromised only by random additive noise is certainly an oversimplification, although there is at least one important area of application - low-dose imaging of unstained frozen-hydrated specimens - in which it appears well founded. Here, the noise arises primarily from electron statistics, and may be eliminated by averaging.

This caveat notwithstanding, a correctly averaged image generally has the great advantage of providing a reproducible, well defined, representation of the specimen, to serve as a basis for inferences concerning its molecular structure. Interpretation is, of course, subject to due caution with respect to possible effects on the molecule's native structure of specimen preparation, radiation

\footnotetext{
* To whom correspondence should be addressed.

Presented at '1990 IUPAB Satellite Congress' 7-10 August 1990.

Palmerston North, New Zealand
}

damage, or the mode of image formation ${ }^{2}$. There are also potential pitfalls in the averaging process itself. For instance, if the molecules are not all intrinsically alike, their global average may be expected to contain artifactual features. Furthermore, if the images are not perfectly aligned, averaging them will result in smearing, thus degrading the finest structural details which the data are potentially capable of yielding. However, as explained below, it is usually possible to avoid such sources of error by careful optimization of the averaging process.

\section{Image averaging in real space and in Fourier space}

With crystalline specimens, image averaging may be performed either in real space or in Fourier space. In real space, it is performed directly on a pixel-by-pixel basis. In the crystal's Fourier transform, the signal is concentrated into discrete, regularly spaced, reflections (the reciprocal lattice), whereas the noise is distributed throughout the diffraction plane. On the reciprocal lattice, therefore, the signal-to-noise ratio is locally very high, and noise may be greatly reduced by multiplying the Fourier transform by a numerical filter function that sets the noisy off-lattice reflections to zero. Finally, the averaged (filtered) image is obtained by inverse Fourier transformation.

These two approaches are formally equivalent: in the Fourier-filtered image, each motif (i.e. repeating element) is a local average in which the weightings depend on the relative sizes of the filter windows and the reciprocal lattice constants ${ }^{3,4}$. In practice, however, there are certain situations in which it is advantageous to use one method rather than the other. For instance, Fourier filtering is 
to be preferred for images that have low contrast or low signal-to-noise ratios, since it is easier in such cases to index the reciprocal lattice in the diffraction pattern than to identify the spacings of the basic repeat in the original image. Furthermore, if two or more crystalline sheets are superposed, the resulting Moiré pattern can be very complex, making it difficult to detect the basic repeat. In the diffraction pattern, the reciprocal lattices of the different crystalline layers are separated from each other (provided that the layers are not in the same orientation), and so each layer may readily be filtered out ${ }^{5}$. On the other hand, with real space averaging, one has greater flexibility in assigning the weights used in averaging unit cells, and it is easier to handle crystalline patches that are small or irregularly shaped. Furthermore, and most importantly for the present discussion, it is easier in real space to compensate for spatial disordering which will otherwise degrade the averaged image.

\section{Spatial disorder in real space and Fourier space}

Crystalline specimens are liable to several different kinds of disorder, such as long-range disorder involving the meandering of lattice-lines, short-range disorder in which each copy of the motif is randomly displaced from its ideal lattice position, liquid-like disorder, etc. ${ }^{6}$. The effects of spatial disorder on the diffraction pattern are that the reciprocal lattice reflections become more diffuse, to an extent that depends on the severity of the disordering. Moreover, this effect increases progressively as one proceeds to higher spatial frequencies (the outermost reflections), and ultimately has the effect of curtailing the radial limit to which these reflections extend, i.e. the effective resolution of the data. In Fourier-based image averaging, diffuse reflections require the use of relatively large windows in the filter function. In consequence, only a limited amount of averaging is achieved and thus only a relatively small improvement in the signal-to-noise ratio (Figure 1). Alternatively, one could include non-uniform 'temperature factors' 7 in the filter function that amplify the outer reciprocal lattice reflections to compensate for their disorder-induced attenuation. However, the precise values that should be used for these temperature factors depend on exact knowledge of the nature of the disorder - information that is not readily available. Moreover, there is no way to restore the high resolution information present in outer reflections that have been completely smeared out. In contrast, with the real-space representation of the image, it is possible to use interpolation procedures of various kinds to correct for spatial disordering. In this way, the correction procedure is tailored to the specific disorder present in each individual specimen.

\section{Imperfectly ordered two-dimensional arrays}

In general, the specifics of the disorder present in a crystal vary stochastically from specimen to specimen. However, crystals of the same kind tend to exhibit the same overall characteristics in their susceptibility to disorder, which, as noted above, may be of several different kinds. In general, this disorder will depend on the rigidity (and homogeneity) of the repeating elements in the crystal, and the strength and regularity of the interactions between them, as well as on the stresses imposed during specimen preparation and imaging. With a view to computational correction, one may distinguish two types of disorder: rigid-body displacements of the motifs from their ideal lattice positions; and distortions in which the motifs undergo plastic deformation. In the former case, the correction strategy preserves the sampling rate of the digital image, while changing the sampling sites to compensate for local displacements; in the latter, the sampling rate is varied to correct for local compression or stretching of the array.

\section{Correlation alignment}

In both cases, the use of cross-correlation and autocorrelation functions ${ }^{8}$ is vital to the correction process. They provide a quantitative, objective, method for precise localization of each copy of the motif. Starting with a reference motif - which may be either a computergenerated model or, more commonly, an image obtained by filtering or averaging the array directly (and consequently, of relatively low resolution) - its crosscorrelation function with the experimental image of the disordered array is calculated. This function contains a set of local maxima whose positions mark the points where the reference motif is optimally aligned with the individual motifs in the array, thus specifying their actual locations*. Individual motifs are then extracted by interpolation, carried out in such a way that the centres of the interpolated motifs coincide with the crosscorrelation maxima. These images are then averaged to provide an improved reference motif, and the cycle repeated.

In the simplest case, only translational offsets are corrected $^{9}$. At the next level of sophistication, orientational changes are also made. To correct for slowly varying changes in orientation, the maxima of the translational cross-correlation function may be used as reference points to re-define the lattice-line directions. Alternatively - and this is the method of choice when relatively large or random orientational offsets are encountered - one may convert both the reference motif and the individual motif of current interest into polar coordinate systems centred on their respective centres of mass, and utilizing equally spaced sampling around a set of concentric annuli. The cross-correlation function is then calculated between corresponding pixels on each annulus. Their relative angular offset is then given by the angle at which this function, summed over all the annuli, is maximized. Successive cycles of translational and orientational refinement are performed, starting with translation, until convergence is achieved. These final

\footnotetext{
* The cross-correlation function is essentially the sum of cross-products between the pixels of one image and the corresponding pixels of the other image, calculated for all possible relative displacements. In the auto-correlation function, the image is combined in this way with itself. To take the simple example of the autocorrelation of a small one-dimensional image $(a, b, c)$, the three cross-products to be considered are $\left(a^{2}+b^{2}+c^{2}\right),(a b+b c+c a)$, and $(a c+b a+c b)$. The maximum clearly occurs when the image is aligned with itself, rather than when cyclically permuted, since$$
\left(a^{2}+b^{2}+c^{2}\right)>/(a b+b c+c a)
$$

on account of the triangle inequality. For larger images in more dimensions, this relationship may be generalized by induction. When seeking to align two images with the same motif, at least one of which is noisy, one looks for their respective signal components $\left(a=a^{\prime}\right)$ to lock on to each other to give the cross-correlation maximum, even in the presence of noise.
} 

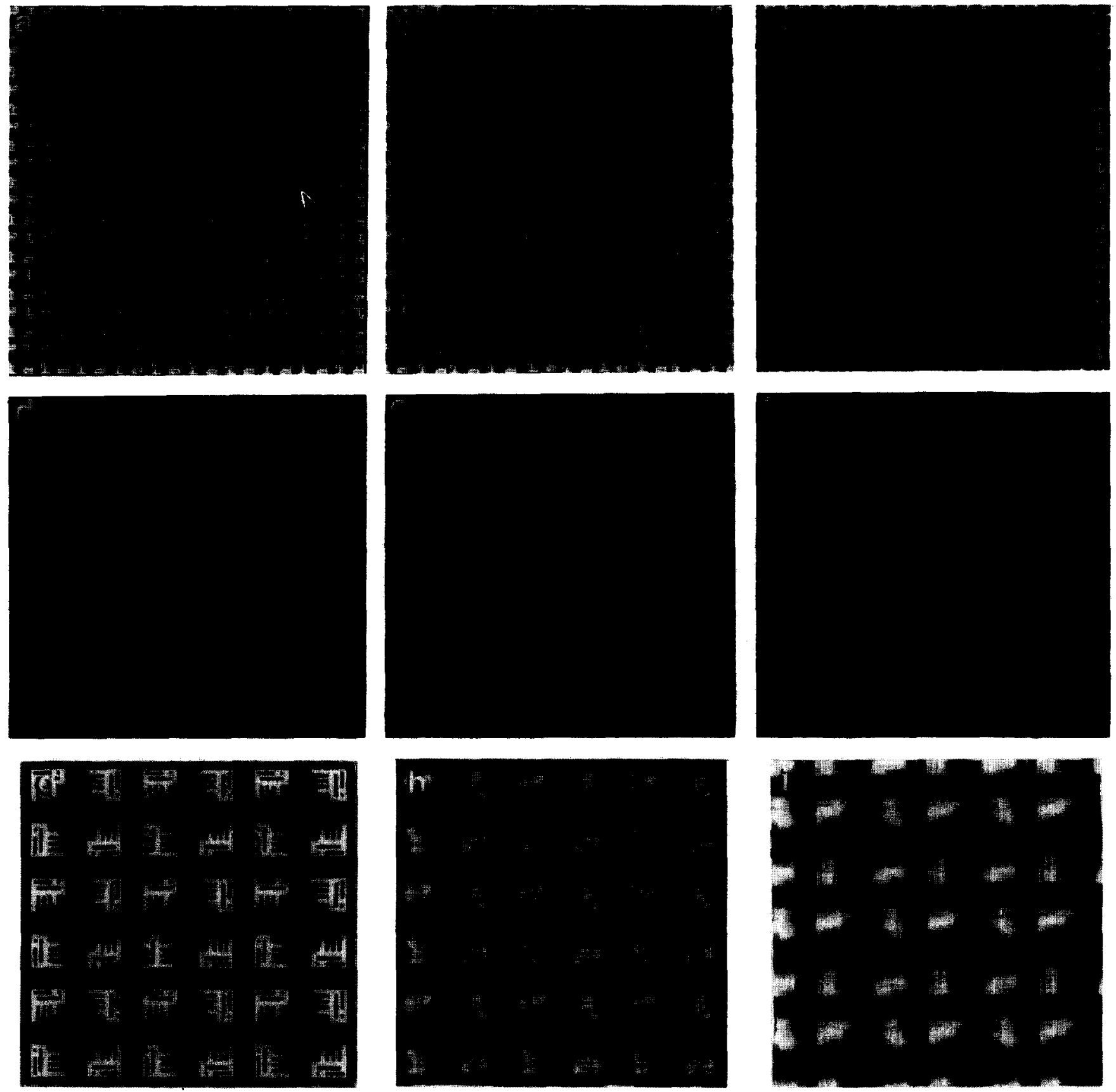

Figure 1 Effects of disorder on the diffraction patterns, and averaging of a two-dimensional crystal (a). In (b), the motifs of the tetragonal lattice were offset from their ideal lattice positions by random displacements of up to 2 pixels $(6 \%$ of the lattice spacing) in both dimensions. In (c), the lattice was distorted by smoothly varying plastic deformation. (d)-(f) show the diffraction patterns (amplitude spectra) of (a)-(c), respectively: (g)-(i) show the corresponding averaged images. Without correction for disorder in (h) and (i), substantial degradation (smearing) of the finer motif details is evident

estimates of the displacement parameters are then used to extract the corrected motif by interpolation from the original image.

\section{Plastic deformations}

When an ordered array is subject to large and non-uniform stresses, it may undergo distortions that are marked by substantial variations in the unit cell dimensions. If the appearance of the distorted crystal indicates that these distortions involve stretching or compression of neighbouring motifs (Figure lc), rather than the decoupling and moving apart of undistorted motifs, it is appropriate to base the correction strategy on the assumption of plastic deformation. One source of data to which this scenario applies is cryo-electron microscopy of frozen-hydrated thin sections, in which compression factors of up to $50 \%$ in the direction of cutting may be encountered ${ }^{10,11}$ (Figure 2). In such cases, the computational procedure is designed to map each unit cell, whose corners are defined by cross-correlation methods, on to an 'ideal' unit cell of fixed dimensions. This may be done either by bilinear interpolation ${ }^{11,12}$, or by interpolation using two-dimensional cubic-splines ${ }^{13}$, or quasi-hermite polynomials ${ }^{14}$ (Figure 3) i.e. a 'rubber sheet' transformation. 

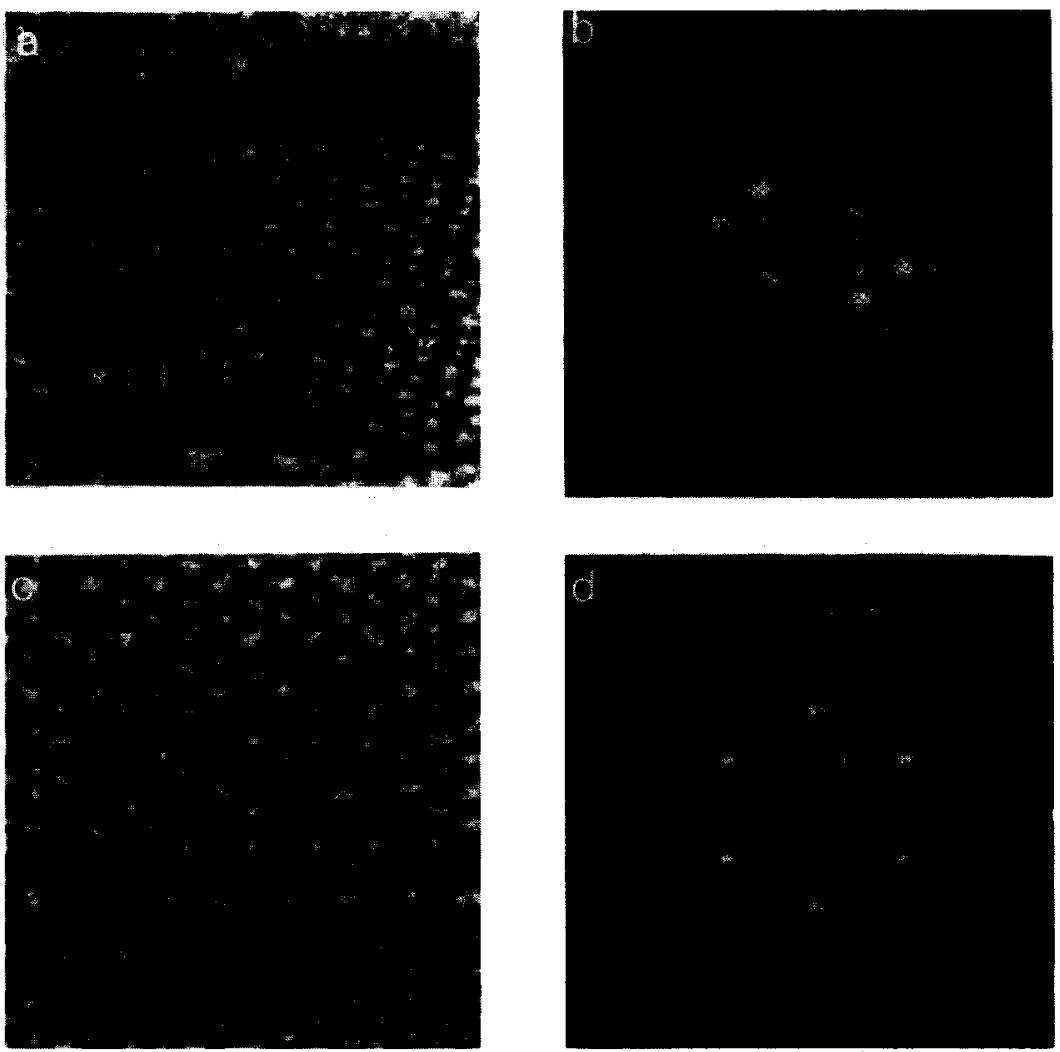

Figure 2 (a) Frozen-hydrated transverse this section of rabbit psoas muscle in rigor, and its diffraction pattern (b). After image restoration, correction for spatial disordering, and consolidation of data from many small patches of myofilament lattice unit cells ${ }^{11}$, the rectified lattice has a considerably richer diffraction pattern. The original pattern (b) shows essentially only the (10) and (11) (upper arrow) reflections, broadened by short-range disorder, with a faint (21) reflection (lower arrow) in one of three possible directions. Moreover, the reciprocal lattice is heavily distorted from hexagonality as a result of sectioning-induced compression. In the rectified diffraction pattern (d), visible periodic reflections extend to the (31) and (32) orders (arrows). The periodicity of the undistorted lattice is $45 \mathrm{~nm}^{11}$
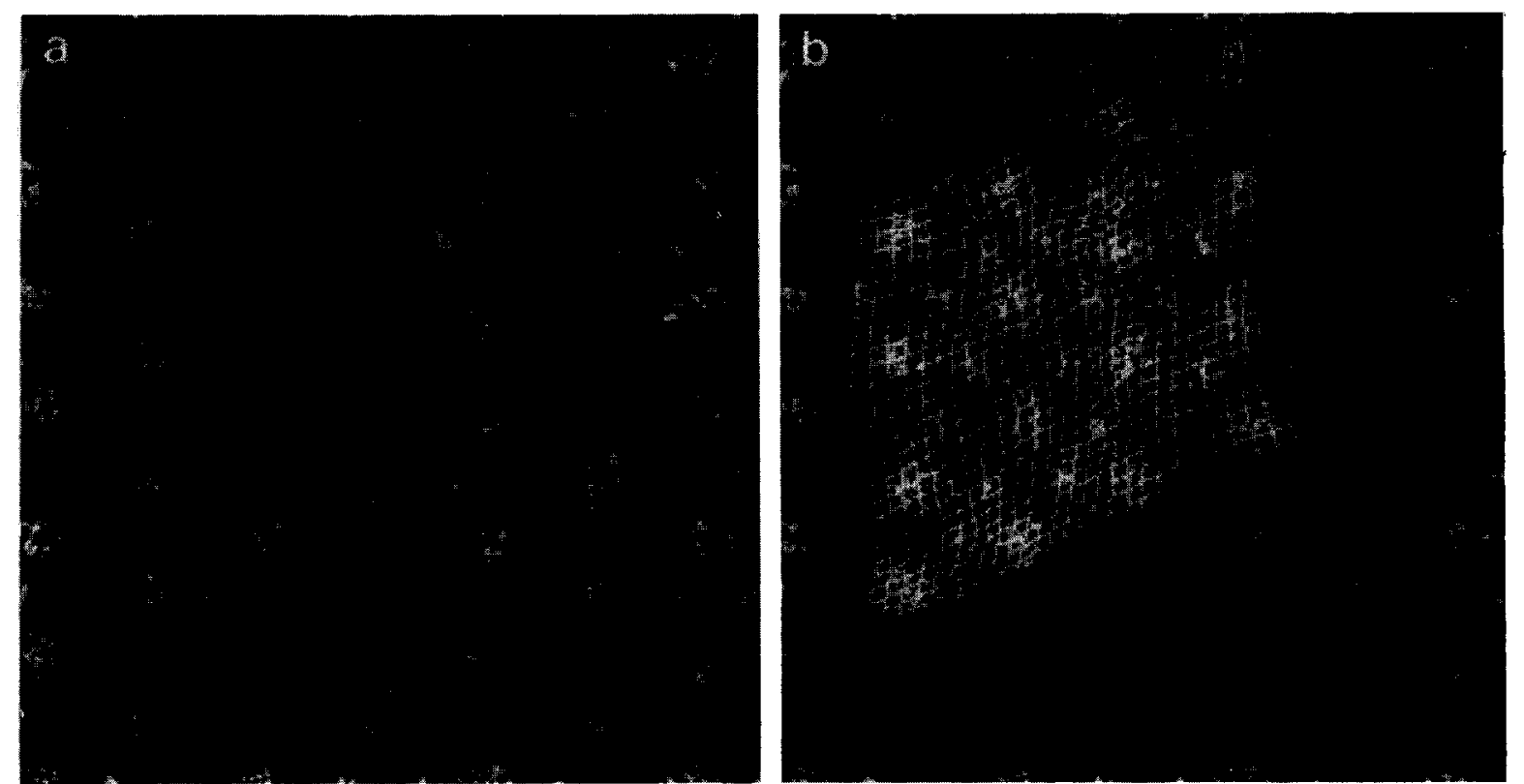

Figure 3 (a) Frozen-hydrated transverse thin section of rabbit psoas muscle in rigor and (b) superimposed sampling grid using quasi-hermite two-dimensional polynomials ${ }^{14}$ to define a correction function

\section{Proofing of unit cells after correction for disorder}

To ensure that the averaged image that is finally obtained is of the best possible quality, i.e. the highest possible resolution, it is helpful to screen the data set to eliminate any anomalous unit cells. An algorithm (OMO = Odd Man Out) has been devised for this purpose ${ }^{15}$. It operates on the principle of maximizing the mutual consistency of the data set. The unit cells are sorted into an ordered list, ranked from most consistent to least consistent with the 
bulk of the data set, and a threshold of acceptability is imposed, based on a statistical criterion. The data thus approved are then averaged with equal weighting. In a more elaborate solution, non-uniform weightings based on rankings in the ordered list may be used ${ }^{16}$.

The above treatment is predicated on the assumption that all unit cells in the crystal are identical, and that any outliers represent unit cells to which some anomalous event has occurred, and are recognizable as such on the basis of random differences from the majority of the data. If, on the other hand, there is reason to suppose that several systematically different classes of unit cell may be present (i.e. one is dealing with a 'mixed' crystal), then the set of unit cells may be analysed by formal classification methods (see below) prior to averaging ${ }^{17,18}$.

\section{Resolution criteria}

In order to assess the improvement attained by computational correction of disorder, or to compare the relative efficacies of several different correction strategies, it is essential to use some quantitative measure of resolution. Traditionally, the resolution of crystalline sheets has been assessed in terms of the spacing of the outermost reciprocal lattice reflection in their diffraction patterns whose intensity is visibly above background, and this criterion may be applied to the data before and after correction. When the final data take the form of a set of individual unit cells, either derived from a single crystal, or scavenged from a number of small crystalline patches $^{19}$, these may be packed together into a crystalline lattice, whose diffraction pattern is then calculated ${ }^{11}$ (cf. Figures $2 b, d$ ). The improvement in resolution may then be assessed by comparing it with the diffraction pattern(s) of the original crystal(s). Prior to packing, it is essential to normalize the data in a uniform way, otherwise dicontinuities in density between neighbouring unit cells may generate artifactual reflections. Alternatively, several quantitative resolution criteria applicable to collections of unit cells have been devised. These include the 'Differential Phase Residual (DPR)',20, the 'Fourier Ring Correlation Coefficient (FFRC)'21, and the 'Spectral Signal-to-Noise Ratio (SSNR) ${ }^{22.23}$ criteria. Since these criteria do not give exactly the same figures for resolution when applied to the same data, it is essential to make consistent use of the same criterion. The SSNR has the appealing feature of being closely related to the traditional diffraction-based resolution criterion discussed above, of which it represents a straightforward generalization $^{22}$

\section{Some practical considerations}

Depending on the complexity of a given analysis, a greater or lesser degree of iteration will, in general, be required in order to achieve the optimal average. The situation may be encountered in the analysis of single particles or individual unit cells that, in some cases, the alternating cycles of translational and orientational alignment do not converge to a stable solution in a reasonable number of cycles (see Refs. 10-12). This is symptomatic of an inadequate signal-to-noise ratio, and such particles are best discarded. The alignment precision and the speed of convergence depend to a considerable degree on the quality of the template (i.e. the reference motif), and for this reason, it is usually desirable to go through the whole analysis several times with progressively refined templates. However, even with a perfect template, the precision with which the correlation maxima are located depends on the signal-to-noise ratio. With very noisy (e.g. very low-dose) images of crystals in which it is reasonable to suspect that long-range disorder is more of a problem than short-range disorder, improved localization of the correlation maxima may be achieved by using a cluster of unit cells, rather than a single unit cell, as template.

\section{Flexible filaments}

Filamentous protein or nucleoprotein polymers are widespread in biology. From electron micrographs, their structural organization may be investigated by indexation of their diffraction patterns (if helical) ${ }^{24}$, computation of their radial density profiles ${ }^{25}$, or full three-dimensional reconstructions ${ }^{26}$. However, macromolecular filaments are seldom absolutely straight, but usually exhibit some degree of curvature, a form of disorder that constrains the scope of any quantitative analysis.

The corrective procedure applied in this situation is to transfer the image into a curvilinear coordinate system specifically tailored to the filament in question (Figure 4). First, a curve is fitted along the particle's centreline, assumed to be a zero-strain path along which distances are preserved. Then, interpolation is used to sample along a family of lines that run perpendicular to the centreline curve, and cross it at equally spaced (one-pixel) intervals. Various alternatives may be considered for parameterizing the centre-line ${ }^{27}$, but cubic splines are a natural choice $^{28-31}$, since a cubic-spline represents the minimumstrain configuration of a stiff, yet flexible, rod affixed to a substrate at the spline's node-points, and this gives a plausible account of how a macromolecular filament adsorbs from solution on to a carbon film prior to electron microscopy ${ }^{28}$

One practical issue in filament straightening is how best to distribute nodes along the centre-line. We are not aware of a rigorous solution to this problem. However, in our experience it is advisable to seek a compromise between node distributions that are too sparse (risking imprecise definition of the axis in the inter-node regions) or too dense (in which case, small lateral offsets between successive node-points may result in anomalous bulges in what should be a smooth curve). For slowly bending filaments, a satisfactory solution is to space the nodes 5-10 pixels apart, and somewhat more closely in regions of relatively high curvature. In a first pass, the operator marks the nodes interactively on a television monitor displaying the particle; their lateral coordinates may subsequently be refined automatically by crosscorrelation methods. Visual inspection of the straightened filament, and subsequent cycles of interactive editing of the nodes is also helpful. A further refinement of this general approach makes it possible to correct for the somewhat more complex disorder associated with variable pitch $^{32}$.

\section{Sparsely sampled filaments}

Scanning transmission electron microscopy (s.t.e.m.) of unstained, frozen-dried, specimens provides data from which radial density profiles may be calculated which are not subject to stain-related ambiguities ${ }^{25}$. Although structural preservation after freeze-drying is not so good as in frozen-hydrated specimens, s.t.e.m. dark-field images 


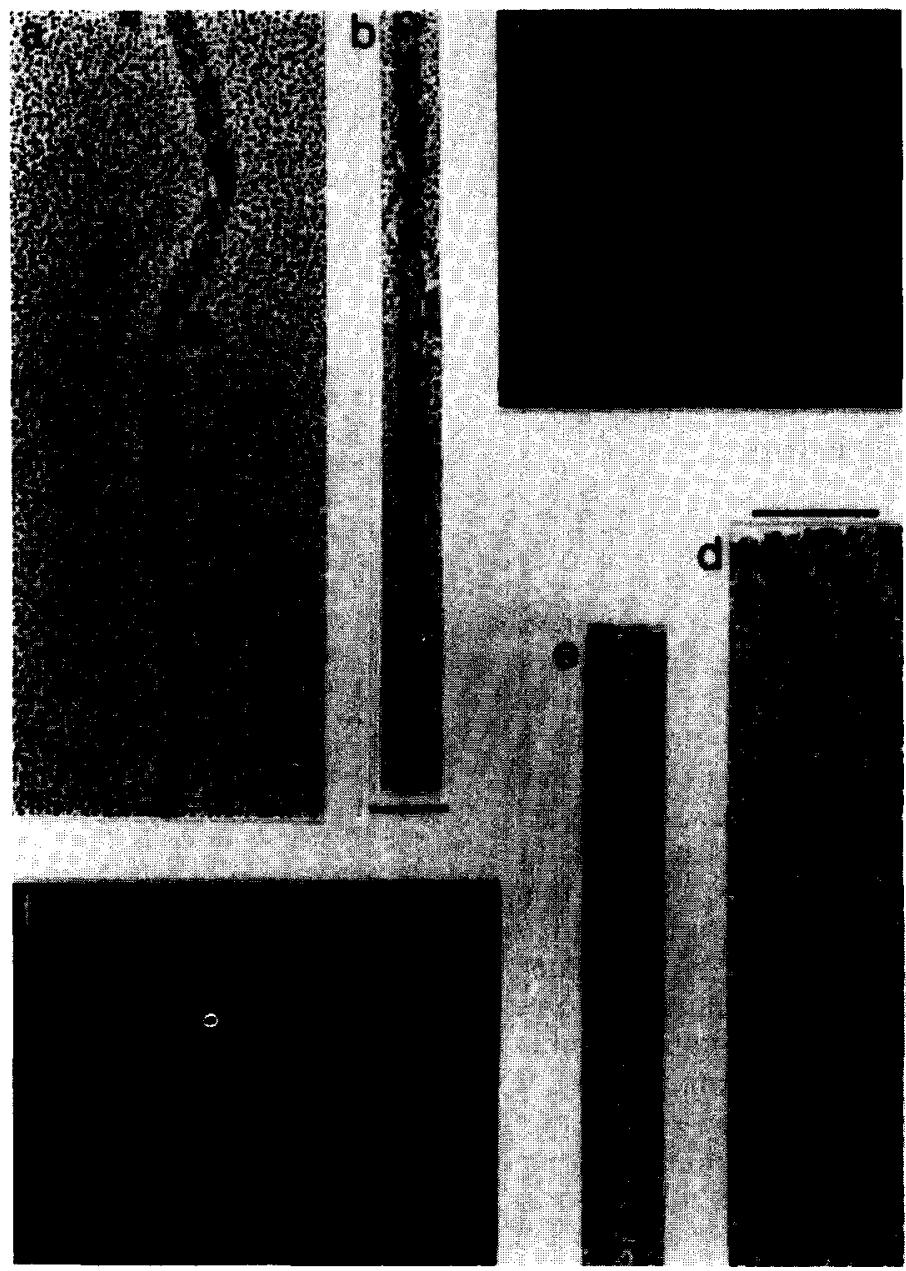

Figure 4 Computational straightening of keratin IF. (a, b) A rotary shadowed murine epidermal keratin IF, before and after computational straightening; (c) computer generated diffraction pattern of (b) which shows a prominent meridional reflection at $(22.7 \mathrm{~nm})^{-1}$; (d, e) negatively stained bovine epidermal keratin IF, before and after straightening and (f) the computer generated diffraction pattern of $(\mathrm{e})$. Arrows indicate an off-meridional reflection at an axial spacing of $\sim(23 \mathrm{~nm})^{-1}$. Bars $=50 \mathrm{~nm}$

have an advantage over conventional t.e.m. bright-field images for the precise localization of edges in that they are not affected by phase contrast-associated interference fringes.

In s.t.e.m., it is customary to sample the specimen relatively sparsely (i.e. at intervals of $1 \mathrm{~nm}, 2 \mathrm{~nm}$ or even $4 \mathrm{~nm})$ with the finely focused $(0.25 \mathrm{~nm})$ s.t.e.m. probe in order to minimize secondary radiation damage. These sampling densities limit the Nyquist frequency (sampling resolution) of the images to $2 \mathrm{~nm}, 4 \mathrm{~nm}$ or $8 \mathrm{~nm}$ respectively. Nevertheless, it is possible to obtain averaged transverse scans of projected density in which the resolution-limiting effects of sparse sampling are overcome by re-sorting the data into a more finely spaced array of bins (Vernier sampling), exploiting the symmetry of the specimen ${ }^{25.33}$. To avoid lateral smearing in these scans, one may confine the axial averaging to short straight segments, and then combine these data by correlation averaging. Alternatively, an algorithm has been developed to compensate for both sparse sampling and bending disorder at the same time ${ }^{34}$.

\section{Oligomeric fibrous proteins}

In addition to fibrous polymers, there are many oligomeric fibrous proteins which exhibit bending disorders, often of an extreme kind, when visualized by electron microscopy, e.g. Ref. 35. These molecules, which usually consist of several polypeptide chains in a parallel association, may also be straightened by cubic spline algorithms ${ }^{36.37}$. Cases affected by particularly tight curvature require the use of two-dimensional cubicsplines. After straightening, the molecules may be classified and averaged ${ }^{36}$. Filtered representations of individual molecules may be obtained hy computationally bending the averaged straightened images back into their original shapes ${ }^{38}$. Furthermore, the curvature profiles generated as a by-product of straightening may be analysed in a systematic way to localize hinge-points or sites of enhanced flexibility ${ }^{36}$.

\section{Averaging individual free-standing particles}

The most extreme case of a disordered crystal is complete dispersal of its repeating elements. Nevertheless, correlation methods, essentially the same as those used to rectify partially ordered arrays ( see above), may be used to align free-standing particles ${ }^{39,40}$. Prior to averaging, these images must be carefully classified into sets of particles that are intrinsically alike. In principle, this process may be expected to be problematic in view of the unlimited range of possible orientations relative to the viewing direction that globular particles might assume. (In a two-dimensional crystal, the interactions between adjacent 
molecules constrain them to the same orientation, so this problem does not arise). However, it transpires that biological macromolecules often tend to adsorb to the support films in a few preferred orientations (e.g. Ref. 41) which has the beneficial effect of greatly limiting the number of classes that must be dealt with. However, sets of dispersed particles have at least one advantage over crystalline arrays. With specimens of the latter type visualized at moderate resolution (say, $2 \mathrm{~nm}$ ), there is frequently ambiguity in how to delineate molecular boundaries in the averaged image, a problem that clearly does not arise with free-standing particles.

\section{Overview and future prospects}

With single-particle averaging, resolutions are routinely achieved that are comparable to those attained for crystals, at least to the limited resolution typical of negatively stained specimens (e.g. $1.5-2.0 \mathrm{~nm}$ ). With a view to extending the structural analysis to higher resolution, the prospects appear to be somewhat brighter for crystalline arrays than for isolated molecules, in the sense that reduction of electron dose to a point that permits survival of high resolution structural detail is more easily accomplished with a crystal. With isolated particles, it is evident that (although this limit is, to some extent, structure-dependent), a certain minimum number of electrons are required to give an image that is statistically well enough defined to allow sufficiently precise correlation alignment and identification of viewing geometry. A major challenge for future studies in this area lies in determining to what extent this inherent limitation may be overcome, for instance, by combining information from high- and low-dose micrographs of the same field.

\section{References}

1 Markham, R. A., Hitchborn, J., Hills, G. J. and Frey, S. Virology $1964,22,342$

2 Misell, D. L. 'Image Analysis, Enhancement, and Interpretation', Elsevier/North Holland Biomedical Press, Amsterdam, 1978

3 Fraser, R. D. B. and Millward, G. R. J. Ultrastruct. Res. 1970, 31, 203

4 Steven, A. C., Aebi, A. and Showe, M. K. J. Mol. Biol. 1976, 102, 373

5 DeRosier, D. J. and Klug, A. J. Mol. Biol. 1972, 65, 469

6 Vainshtein, B. K. 'Diffraction of X-Rays by Chain Molecules', Elsevier Publishing Co., Amsterdam, 1966

7 Stout, G. H. and Jensen, L. H. 'X-Ray Structure Determination' MacMillan, New York, 1968

8 Frank, J. in "Advanced Techniques in Biological Electron Microscopy` (ed. J. K. Koehler), Springer Verlag, 1973, p 213
9 Crepeau, R. H. and Fram, E. K. Ultramicroscopy 1981, 6, 7

10 McDowall, A. W., Hoffmann, W., Lepault, J., Adrian, M. and Dubochet, J. J. Mol. Biol. 1984, 178, 105

11 Trus, B. L., Steven, A. C., McDowall, A. W., Unser, M., Dubochet, J. and Podolsky, R. J. Biophys. J. 1989, 55, 713

12 Crowther, R. A. and Sleytr, U. B. J. Ultrastruct. Res. 1977, 58, 41

13 Henderson, R., Baldwin, J. M., Downing, K. H., Lepault, J. and Zemlin, F. Ultramicroscopy 1986, 19, 147

14 Unser, M., Trus, B. L. and Eden, M. Signal Processing 1989, 17, 191

15 Unser, M., Steven, A. C. and Trus, B. L. Ultramicroscopy 1986, 19,337

16 Unser, M. and Eden, M. IEEE Trans. Acoust. Speech Signal Proc. 1990, ASSP-38, 890

17 Frank, J., Chiu, W. and Degn, L. Ultramicroscopy 1988, 26, 345

18 Sass, H. J., Bueldt, G., Beckmann, E., Zemlin, F., Van Heel, M., Zeitler, E., Rosenbusch, J. P., Dorset, D. L. and Massalski, A. J. Mol. Biol. 1989, 209, 171

19 Steven, A. C., Roberts, C. R., Hay, J., Bisher, M. E., Pun, T. and Trus, B. L. J. Virol. 1986, 57, 578

20 Frank, J. Ultramicroscopy 1975, 1, 159

21 Saxton, W. O. and Baumeister, W. J. Micros. 1982, 127, 127

22 Unser, M., Trus, B. L. and Steven, A. C. Ultramicroscopy 1987. 23, 39

23 Unser, M., Trus, B. L., Frank, J. and Steven, A. C. Ultramicroscopy 1989, 30, 429

24 Fraser, R. D. B. and MacRae, T. P. 'Conformation in Fibrous Proteins', Academic Press, New York, 1973

25 Steven, A. C., Hainfeld, J. F., Trus, B. L., Steinert, P. M. and Wall, J. S. Proc. Natl. Acad. Sci. USA 1984, 81, 6363

26 DeRosier, D. J. and Moore, P. B. J. Mol. Biol. 1970, 52, 355

27 Fraser, R. D. B., MacRae, T. P., Suzuki, E. and Davey, C. L. J. Microse. 1976, 108, 343

28 Steven, A. C., Stall, R., Steinert, P. M. and Trus, B. L. in 'Electron Microscopy and Alzheimer's Disease', (ed. J. Metuzals) San Francisco Press, San Francisco, 1986, p 31

29 Egelman, E. Ultramicroscopy 1986, 19, 367

30 Steven, A. C. in 'Intermediate Filament Structure: Diversity, Polymorphism and Analogy to Myosin, (eds. R. D. Goldman and P. M. Steinert) Pergamon Publishing Corp., 1990, p 233

31 Hutchinson, G., Tichelaar, W., Weiss, H. and Leonard, K. J. Struct. Biol. 1990, 103, 75

32 Bluemke, D. A., Carragher, B. and Josephs, R. Ultramicroscopy 1988, 266, 255

33 Steven, A. C., Simpson, T. A., Trus, B. L., Furcinitti, P. S., Hainfeld, J. F. and Wall, J. S. Ann. NY Acad. Sci. 1986, 483, 188

34 Day, L., Marzec, C. J., Reisberg, S. A. and Casadevall, A. Annu. Rev. Biophys. Biophys. Chem. 1988, 17, 509; Reisberg, S. PhD thesis, New York University

35 Aebi, U. and Engel, J. (eds) 'Cytoskeletal and Extracellular Proteins: Structure, Interactions, and Assembly', Springer Verlag, Berlin, 1989

36 Fraser, R. D. B., Furlong, D. B., Trus, B. L., Nibert, M. L., Fields, B. N. and Steven, A. C. J. Virol. 1990, 64, 2990

37 Steven, A. C., Trus, B. L., Maizel, J. V., Unser, M., Parry, D. A. D., Wall, J. S., Hainfeld, J. F. and Studier, F. W. J. Mol. Biol. $1988,200,351$

38 Koesis, E., Trus, B. L., Steer, C. J., Bisher, M. E. and Steven, A. C. (In preparation)

39 Van Heel, M. and Frank, J. Ultramicroscopy 1981, 6, 187

40 Unser, M., Trus, B. L. and Steven, A. C. Ultramicroscopy 1989, 30, 299

41 Van Heel, M. and Stoeffler-Meilicke, M. EMBO J. 1985, 4, 2389 